\title{
BMJ Open Electroacupuncture for treating insomnia in patients with cancer: a study protocol for a randomised pilot clinical trial
}

\author{
Mikyung Kim, ${ }^{1}$ Jung-Eun Kim, ${ }^{1}$ Hye-Yoon Lee, ${ }^{1}$ Ae-Ran Kim, ${ }^{1}$ Hyo-Ju Park, ${ }^{1}$ \\ O-Jin Kwon, ${ }^{1}$ Bo-Kyung Kim, ${ }^{2}$ Jung Hyo Cho, ${ }^{3}$ Joo-Hee Kim ${ }^{1}$
}

To cite: Kim M,

Kim J-E, Lee H-Y, et al.

Electroacupuncture for treating insomnia in patients with cancer: a study protocol for a randomised pilot clinical trial. BMJ Open 2017;7:e016269. doi:10.1136/ bmjopen-2017-016269

- Prepublication history and additional material are available. To view these files please visit the journal online (http://dx.doi. org/10.1136/bmjopen-2017016269).

Received 3 February 2017 Revised 13 May 2017 Accepted 24 May 2017

\section{(a) CrossMark}

${ }^{1}$ Clinical Research Division, Korea Institute of Oriental Medicine, Daejeon, Republic of Korea

2Department of Neuropsychiatry, College of Oriental Medicine,

Dong-eui University, Busan, Republic of Korea

${ }^{3}$ Department of Internal

Medicine, College of Traditional

Korean Medicine, Daejeon

University, Daejeon, Republic of Korea

Correspondence to

Dr Joo-Hee Kim;

jhkim714v@gmail.com

\section{ABSTRACT}

Introduction Although insomnia is one of the most prevalent and disturbing symptoms among patients with cancer, it has not been properly managed.

Electroacupuncture (EA) has received attention as a promising intervention for insomnia, and a few previous studies have reported that this intervention may be beneficial for treating insomnia in patients with cancer. The aim of this pilot study is to explore the feasibility and preliminary effectiveness of EA on the sleep disturbance of patients with cancer with insomnia using a subjective method, patient-reported questionnaires and an objective tool, actigraphy, to measure the quality of sleep.

Methods and analysis This is a study protocol for a randomised, three-arm, multicentre, pilot clinical trial. A total of 45 patients with cancer who have continuous insomnia related to cancer treatment or cancer itself will be randomly allocated to an EA group, sham EA group or usual care group in equal proportions. The EA group will receive 10 sessions of EA treatment over 4 weeks. The sham EA group will receive sham EA at non-acupoints using non-penetrating Streitberger acupuncture needles with mock EA. The usual care group will not receive EA treatment. All participants will be provided a brochure on the management of sleep disorders regardless of their group assignment. The primary outcome measure is the mean change in the insomnia severity index from the baseline to week 5 . Information related to sleep quality will also be obtained through the Pittsburgh Sleep Quality Index, a sleep diary and actigraphy. Participants will complete the trial by visiting the research centre at week 9 for follow-up assessment.

Ethics and dissemination This study protocol was approved by the institutional review boards of each research centre. Written informed consent will be obtained from all participants. The result of this study will be published in peer-reviewed journals or presented at academic conferences.

Trial registration number KCT0002162; Pre-results.

\section{INTRODUCTION}

Insomnia is one of the most common afflictions among the general population, and the prevalence of insomnia is known to be twice
Strengths and limitations of this study

- This randomised, multicentre clinical trial is the first study in South Korea exploring the effect and safety of electroacupuncture as a treatment for sleep disturbance in patients with cancer with insomnia. In addition to patient-reported outcomes, the study will use actigraphy to acquire objective outcomes reflecting the quality of sleep and measure the levels of cortisol and melatonin in saliva as biomarkers reflecting the circadian rhythm

- Polysomnography needs to be included in future research to investigate sleep architecture and the various causes of sleep disorders.

- Because of the unique nature of electroacupuncture and the design of this study, which includes a usual care group, it is impossible to completely blind the practitioners and all participants.

- The study adopts non-penetrating needling at nonacupoints as a sham comparator for acupuncture; however, a perfect placebo intervention for real acupuncture that exerts no physiological effects while maintaining patient blinding has not been developed yet.

- The sample size of this pilot study is 15 per each group, which may be underpowered to draw a definitive conclusion of the effectiveness of the intervention.

as high as in patients with cancer compared with healthy adults. ${ }^{1}$ Complex interactions of various factors such as adverse reactions to chemotherapy, ${ }^{1}$ circadian disruption, ${ }^{2}$ inflammation $^{3}$ and tumour growth ${ }^{3}$ seem to be the reasons why patients with cancer are especially susceptible to insomnia. Insomnia has a profound effect on the overall quality of life, ${ }^{4}$ energy, ${ }^{5}$ emotion, ${ }^{6}$ memory ${ }^{7}$ and immune system ${ }^{8}$ of patients with cancer. In addition, insomnia is also known to be associated with decreased survival $^{9}$ and tumour worsening. ${ }^{10}$ Although insomnia is such a common and important problem 
among patients with cancer, it is not easily cured or well managed. ${ }^{11}$

The most commonly used therapy for the management of insomnia in patients with cancer is pharmacotherapy. ${ }^{12}$ According to Moore et al, approximately half of patients with breast cancer are prescribed sleeping pills. ${ }^{13}$ Longterm use of sleeping pills is known to be associated with continued sleep disturbance, decreased ability to perform daily activities, memory impairment, traffic accidents, falls, etc. ${ }^{12}$ Thus, pharmacotherapy should be used only for a short-term period; however, approximately $30 \%$ of patients with cancer are receiving sleeping pills even 1 year after the end of chemotherapy. ${ }^{13}$

In addition to pharmacotherapy, cognitive behavioural therapy has been recommended as a standard therapy for the improvement of insomnia. ${ }^{3}$ However, there are some limitations to this therapy in that the compliance rate to the therapy is low because the therapeutic response does not appear immediately, and patient access to the therapy is low due to the high cost of the therapy. ${ }^{14}$ Moreover, experienced therapists are rare, and the effect of therapy varies widely depending on the proficiency of the therapist. $^{14}$

For this reason, there is a growing demand for complementary medicine (CAM) in the field of sleep management. Acupuncture is one of the most commonly used therapeutic interventions in CAM. Acupuncture is a treatment that punctures and stimulates the skin at acupoints using specialised needles. Electroacupuncture (EA) refers to a method that supplies a constant physical stimulus to acupoints through the inserted acupuncture needle connected to a microcurrent stimulator.

Previous systematic reviews on the effect of acupuncture for insomnia have revealed that acupuncture is significantly more effective to improve sleep disturbance in insomniacs than no treatment ${ }^{15}$ and sham acupuncture. ${ }^{15-17}$ Insomnia has also been reported to be as effective as pharmacotherapy ${ }^{15}$ or even superior to sleeping pills ${ }^{15} 17$ and provides additional benefit to patients who are undergoing pharmacotherapy to improve insomnia. ${ }^{1516}$

The effect of acupuncture for the improvement of sleep disorders has also been identified in studies targeting patients with cancer. A survey conducted at a major cancer centre in the USA showed that acupuncture significantly improved the quality of sleep in patients with cancer. ${ }^{18}$ Garland et al also determined that 8-week EA was more effective than pharmacotherapy to attenuate sleep disturbance from a recently published randomised controlled trial (RCT) including patients with breast cancer suffering from hot flush. ${ }^{19}$ Choi $e$ t al systematically investigated the clinical studies on the effect of acupuncture for insomnia in patients with cancer and indicated that acupuncture may be superior to sham acupuncture or pharmacotherapy ${ }^{20}$ However, these authors could not draw any firm conclusions because the level of evidence in the included studies was low overall, the effect size of the studies was too small to confirm their clinical significance and there were few studies strictly designed and performed. ${ }^{20}$

Subjective measurement tools such as self-reported questionnaires or sleep diaries are the most commonly used type of outcome measures for the assessment of sleep quality. This is because these tools are relatively simple to measure and have the advantage of not interfering with daily life or sleeping; however, one limitation of this approach is that the results of these measurements are entirely up to the decisions of participants and are unable to objectively measure the pattern of sleep. ${ }^{21}$ Polysomnography and actigraphy are the two main methods to objectively measure sleep quality. ${ }^{21}$

Polysomnography is a gold standard method for the objective measurement of sleep ${ }^{321}$ because it can demonstrate the sleep structure and the cause of sleep disorders in various ways including electroencephalogram, electromyogram, ECG, respiration and oxygen saturation. ${ }^{22}$ However, one disadvantage is that polysomnography requires an expensive device and an expert capable of interpreting the results. ${ }^{21}$

To overcome these shortcomings, actigraphy has been investigated..$^{23}$ Actigraphy is a non-invasive means of measuring the motion of the body using an actigraph, a piezoelectric accelerometer. ${ }^{23}$ Actigraphy has been widely used in the field of sleep research because it can detect sleep, activity and movement without disturbing the daily lives of the subjects who are only required to wear a small, watch-shaped device on the wrist. ${ }^{23}$ Studies on the similarities and differences between the sleep quality measured by polysomnography, actigraphy and subjective assessment tools have been published. ${ }^{23}$ It is recommended to use both objective and subjective measurement methods to assess sleep quality in the field of sleep research. ${ }^{24}$

A few acupuncture researchers have reported the positive effect of acupuncture using objective measurement tools such as polysomnography ${ }^{25-27}$ or actigraphy. ${ }^{28}{ }^{29}$ However, there remain conflicting results regarding whether acupuncture has the same effect on both subjective and objective aspects of sleep. Some researchers argued that the effect of acupuncture was approved via both objective and subjective measurement tools ${ }^{25-27}$ while others reported that acupuncture seemed to affect the subjective outcomes only, not the result of polysomnography ${ }^{30}{ }^{31}$ or actigraphy. ${ }^{32-34}$

The aim of this pilot study is to explore the feasibility and preliminary effectiveness of EA on the sleep disturbance of patients with cancer with insomnia using both subjective questionnaires and objective actigraphy.

\section{METHODS AND ANALYSIS \\ Study design}

This is a study protocol for a randomised, three-arm, multicentre, pilot clinical trial. Forty-five patients with cancer with insomnia will be randomly assigned to an EA group, sham EA group or usual care group at a 1:1:1 ratio. The schedule of enrolment, interventions and assessments is 
Table 1 Schedule of enrolment, interventions and assessments

\section{Study period}

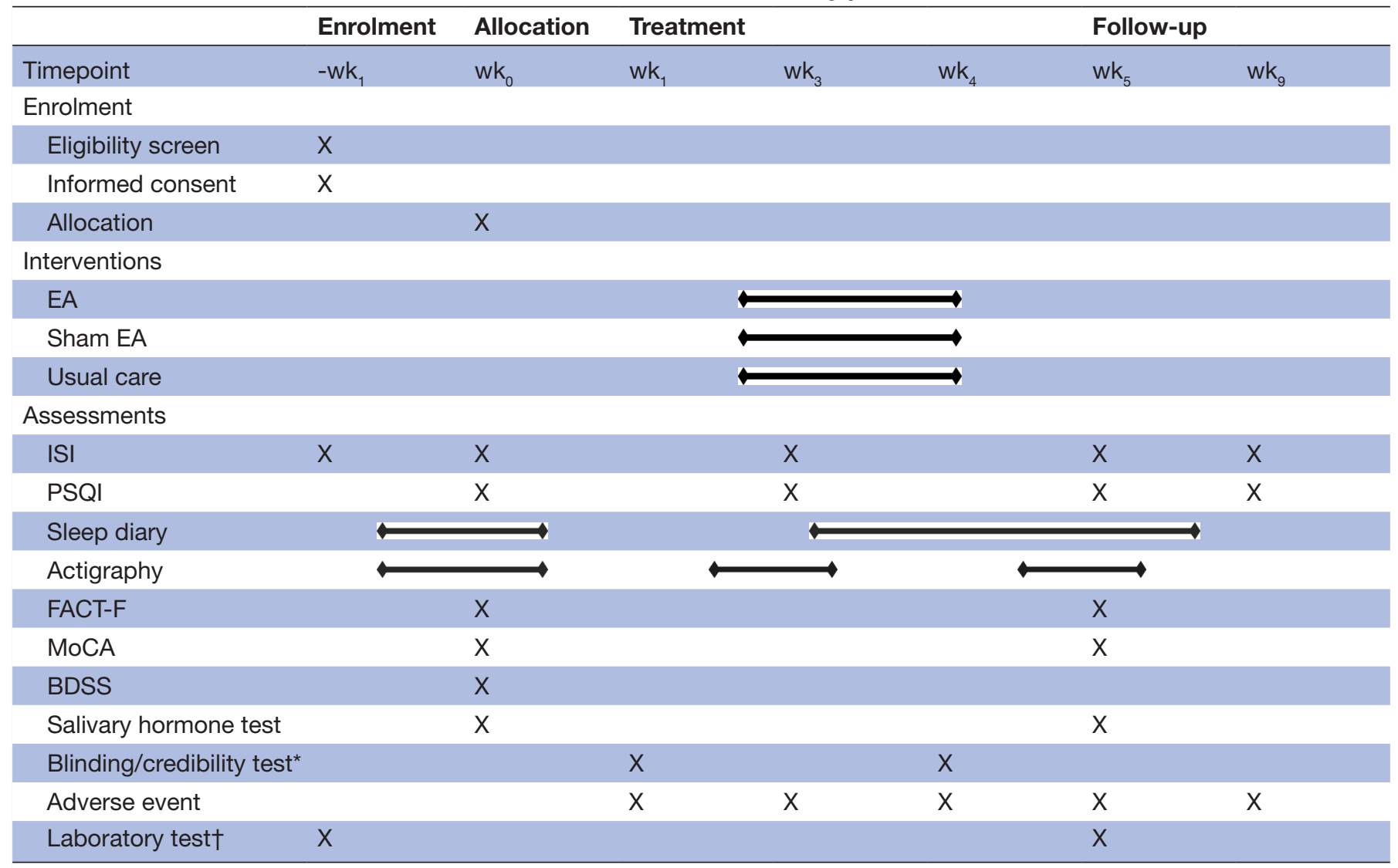

*Only EA and sham EA groups will undergo blinding and credibility tests.

†The laboratory test includes complete blood count and differential count, absolute neutrophil count, aspartate aminotransferase, alanine aminotransferase, total bilirubin, blood urea nitrogen, creatinine, albumin, erythrocyte sedimentation rate, thyroid-stimulating hormone, free thyroxine and human chorionic gonadotropin urine test (only for women in their childbearing years at the screening visit).

BDSS, Blood Deficiency Scoring System; EA, electroacupuncture; FACT-F, Functional Assessment of Cancer Therapy-Fatigue; ISI, Insomnia Severity Inventory; MoCA, The Montreal Cognitive Assessment; PSQI, Pittsburgh Sleep Quality Index.

summarised in table 1 , and the flow diagram of the study is presented in figure 1 .

\section{Recruitment}

Two clinical research centres in South Korea will conduct this trial: Daejeon Oriental Hospital of Daejeon University and Dong-eui University Korean Medical Hospital in Busan. We will do our best to secure enough participants in this study using online and offline advertisement boards inside and outside the hospitals and by releasing flyers to daily local newspapers.

\section{Inclusion criteria}

1. Patients aged 19 years or over but under 80 years;

2. An Eastern Cooperative Oncology Group performance status $\leq 2$;

3. Cessation of cancer-related treatments (eg, surgery, radiotherapy, chemotherapy, immunotherapy) at least 12 weeks before the trial (ongoing hormone therapy, which must have been initiated at least 3 weeks prior to enrolment, is allowed);
4. Continuous insomnia related to cancer treatment or cancer itself for at least 3 months, with fulfilment of the Diagnostic and Statistical Manual of Mental Disorders, Fifth Edition diagnostic criteria for insomnia disorder;

5. Total score in the Insomnia Severity Index $($ ISI $) \geq 15$ points;

6. Willingness to participate in the trial and having provided written consent;

\section{Exclusion criteria}

1. Having experienced the current level of sleep disorder prior to the diagnosis of cancer;

2. Severe anaemia (platelet count $<60000 / \mu \mathrm{L}$, haemoglobin $<8 \mathrm{~g} / \mathrm{dL}$ or absolute neutrophil count $<1000 / \mu \mathrm{L})$;

3. A diagnosis of major depressive disorder, anxiety disorder, panic disorder or other psychiatric disorder; caffeine, alcohol or drug addiction; or a subscale of either anxiety or depression in Hamilton Anxiety and Depression Scale score $\geq 11$ points; 


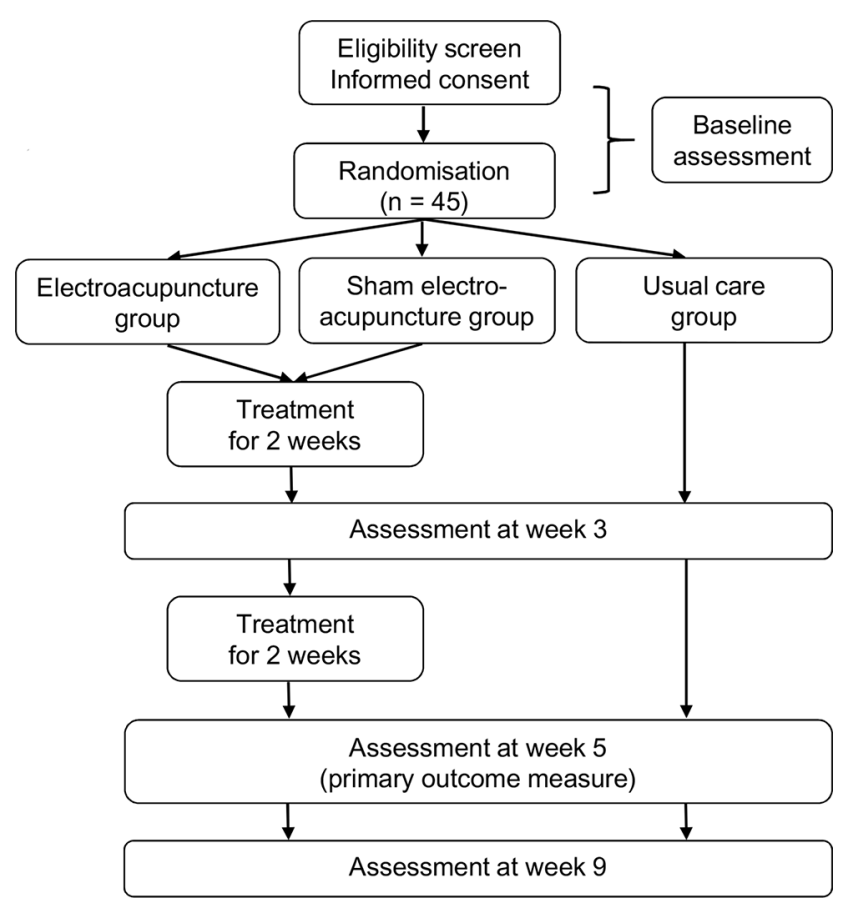

Figure 1 Flow diagram of the study.

4. Level of cancer pain measured by the numeric rating scale $\geq 4$

5. An estimated life expectancy of 6 months or less;

6. A plan for surgery, chemotherapy or radiotherapy during the study;

7. A recent change of regular medication to alleviate insomnia within 4 weeks of the beginning of the trial;

8. Having taken sleeping pills as required within 2 weeks of the beginning of the trial;

9. Having undergone Korean medical treatment (eg, acupuncture, moxibustion, cupping, herbal medicine) within 4 weeks of the beginning of the trial;

10. Initiation or a change in dietary supplement regimen or non-pharmacological therapies (eg, cognitive behavioural therapy, exercise, etc) for alleviating insomnia during the trial or within 4 weeks of the beginning of the trial;

11. Working shifts or changes in day/night work schedule that could impact circadian rhythm;

12. Suffering from pain severe enough to cause sleep disturbance or presence of any disease that could cause insomnia;

13. Taking haemostatic agents (eg, Greenmono, Advate, Monoclate-P, Facnyne, BeneFix) because of haemostatic disorders;

14. Abnormal findings in thyroid function test (abnormal level of free thyroxine (free T4) and thyroid-stimulating hormone $(\mathrm{TSH})<0.1 \mu \mathrm{IU} / \mathrm{mL}$ or $\mathrm{TSH}>5.1 \mu \mathrm{IU} / \mathrm{mL}$ );

15. History of hypersensitivity reactions to acupuncture or inability to cooperate with acupuncture therapy;
16. The presence of implants that could interfere with EA or a history of hypersensitivity reactions to electrostimulation;

17. Pregnancy, lactation or planning to become pregnant;

18. Having participated in other clinical trials within 4 weeks of the beginning of the trial;

19. Difficulty complying with the study protocol;

\section{Randomisation and allocation concealment}

Fifteen subjects will be allocated to each group according to a randomisation schedule generated by an independent statistician using SASV.9.4. A stratified randomisation method will be used with the research centre and the standard therapies for insomnia (regular sleep medications or cognitive behavioural therapy for insomnia) as stratification factors. The statistician will seal the randomisation codes in sequentially numbered opaque envelopes and send them to the research centres. These envelopes will be kept in double-locked cabinets at the centres. Each envelope will be opened by a practitioner to assign the participant to one of the three groups after acquiring informed consent and eligibility screening.

\section{Blinding}

Participant blinding will be limited to the participants in the EA and sham EA groups. Complete participant blinding is not possible because the subjects in the usual care group cannot help but notice their allocated group. Practitioner blinding cannot be achieved because of the unique nature of EA treatment. The assessor blinding will be maintained by separating the staff members who perform EA and who measure the outcomes.

\section{Interventions}

All participants of this trial will be educated with a brochure on the management of sleep disorders regardless of the assigned groups.

\section{EA group}

Participants in the EA group will receive acupuncture treatment at GV20, EX-HN3, bilateral HT7, PC6, BL63 and KI4 using sterilised stainless steel acupuncture needles (Asiamed, $0.25 \times 25 \mathrm{~mm}$ ) (figure 2 ). In addition to these 10 acupoints, up to 4 more points can be optionally added according to the symptoms of the patients. If participants with lymphatic oedema refuse to be treated with the acupoints on the affected site, the acupoints near the oedema site can be omitted. After inducing deqi by twisting the acupuncture needles, electric stimulation using an EA device (ES-160; Ito Co, Tokyo, Japan) will be applied with a $4 \mathrm{~Hz}$ frequency, at an intensity that the participant can notice but feel comfortable with. The EA treatment will be performed for $30 \mathrm{~min}$ per time, with a total of 10 times over 4 weeks. This regimen was decided by consensus among experts of traditional Korean medicine based on previous research. ${ }^{15-20}$ 25-29 313234 Details of the EA treatment based on the standards for reporting interventions in clinical trials of the acupuncture checklist are tabulated in online supplementary file 1 . 

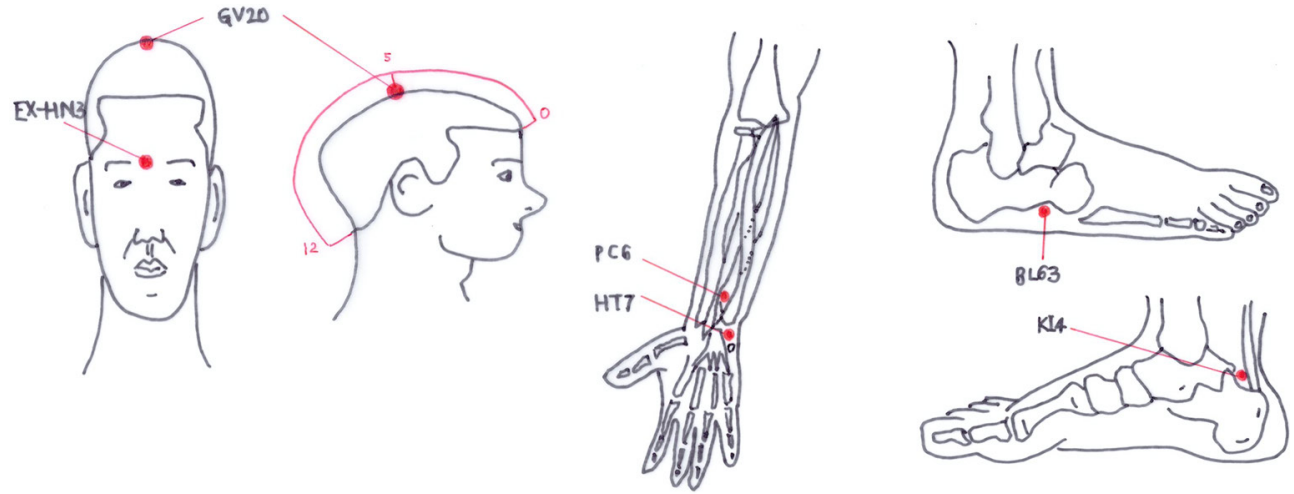

Figure 2 The points used for the electroacupuncture group.

\section{Sham EA group}

Participants in the sham EA group will receive sham acupuncture using non-penetrating Streitberger acupuncture needles (Asiamed). Unlike the real acupuncture needle that is inserted into the skin, the tip of the Streitberger acupuncture needle is so blunt that it cannot penetrate the skin. The needles will be fixed by medical skin tape on the skin at non-acupoints unrelated to the management of sleep disorders in Korean traditional medicine as follows: upper limb $1(1 \mathrm{~cm}$ lateral and $5 \mathrm{~cm}$ proximal points from cubital creases of bilateral arms), upper limb 2 (2 $\mathrm{cm}$ above the upper limb 1 point), lower limb $1(1.5 \mathrm{~cm}$ above the depression at the midpoint of the upper border of the bilateral patella), lower limb 2 (area $1 / 3$ above the medial part of the bilateral tibia) and lower limb 3 (1.5 cm above the lower limb 2 point). The same EA device will be applied to the Streitberger acupuncture needles, with an identical beeping sound and light signals as for the EA group. However, the electric current will not be delivered.

\section{Usual care group}

Participants in the usual care group will not receive EA treatment. These subjects will maintain the usual treatment and self-care but cannot begin any additional treatment to improve their insomnia during the study period.

\section{Prohibited and permitted concomitant treatment}

The participants will be prohibited from any Korean traditional medical treatment such as acupuncture, moxibustion, cupping or herbal drugs to attenuate their insomnia symptoms except for the EA provided by the trial. If the participants remained on regular medication to improve insomnia at least 4 weeks prior to the beginning of the trial, they will be allowed to maintain the medication. However, if the type or dosage of the sleeping pill is changed during the trial, the subjects will be withdrawn from the study. If the subjects are receiving standard non-pharmacological treatment, such as cognitive behavioural therapy, or taking any dietary supplements to attenuate insomnia from at least 4 weeks prior to the beginning of the trial, they will be allowed to maintain these treatments. However, their regimens cannot be changed during the trial.

The participants will be trained to report all treatments newly received after the beginning of the trial to the staff members, and their treatment history will be recorded on the case report form (CRF). When participants are found to have received a prohibited treatment during the study period, they will be withdrawn from the trial. Despite withdrawal from the trial, the recordings of the participants will continue if the subjects have already randomised. To realise the intention-to-treat principle, the data from the withdrawn participants will also be included in the analysis set and recorded in the results.

\section{Outcomes}

Study feasibility outcomes

To see whether a full-scale randomised clinical trial for this issue is feasible, the recruitment rate and completion rate will be calculated. The overall rate of adherence to the planned intervention will also be ascertained.

\section{Primary outcome measure}

The primary outcome measure of this study will be the mean change in the ISI from the baseline to the end of the 4 week intervention. The ISI is a seven-item questionnaire devised to diagnose and assess the severity of the insomnia. The total score of the ISI ranges from 0 to 28 points, and it can categorise insomniacs according to scores of $0-7$ (no clinically significant insomnia), 8-14 (subthreshold insomnia), 15-21 (clinical insomnia, moderate severity) and 22-28 (clinical insomnia, severe). ${ }^{35}$ The Korean version of the ISI validated by Cho et al will be used in this study. ${ }^{36}$

\section{Secondary outcome measures}

The secondary outcome measures of this study will include the mean changes in the total score of the ISI from baseline to week 3 and week 9 and changes in the scores of the Pittsburgh Sleep Quality Index (PSQI), Functional Assessment of Cancer Therapy-Fatigue (FACT-F) and Montreal Cognitive Assessment (MoCA); the level of the salivary hormones including cortisol and melatonin; and 
the quality of sleep measured by a sleep diary and actigraphy before and after the intervention.

The PSQI is a self-reported questionnaire to assess the quality of sleep over the past month. This scale comprises seven components including subjective sleep quality, sleep latency, sleep duration, sleep efficiency, sleep disturbances, use of sleeping pills and daytime dysfunction. The global PSQI score ranges from 0 to 21, and a higher score represents poorer quality of sleep. ${ }^{37}$ The Korean version of the PSQI validated by Sohn et al will be used in this study. ${ }^{38}$

The change of the global score in the PSQI will be assessed at baseline, week 3 , week 5 and week 9 .

The FACT-F is a questionnaire devised to determine the level of fatigue of patients with cancer. This instrument consists of the FACT-general $(\mathrm{G})$ and the fatigue subscale. The FACT-G is composed of 27 items in four domains including physical well-being, social/family wellbeing, emotional well-being and functional well-being. The fatigue subscale has 13 items assessing the level of fatigue. The total score of the FACT-F ranges from 0 to 160 , where higher points denote a higher level of fatigue that disturbs the daily life of the patients. ${ }^{39}$ Lee et al validated the Korean version of the FACT-G. ${ }^{40}$ Jeong et al have translated and validated the Korean version of the fatigue subscale and also used it with Lee's Korean version of the FACT-G among patients with cancer-related fatigue. ${ }^{41}$ In other words, the Korean version of the complete FACT-F has been used to measure the level of fatigue among patients with cancer in Korea. ${ }^{41}$ FACT-F scores will be assessed at baseline and week 5 .

The MoCA is a one-page, 30-point test originally devised to screen for mild cognitive impairment in the geriatric population and can also be applied to evaluate the level of cognition of cancer survivors. ${ }^{42}$ The validated Korean version of the MoCA will be used in this study. ${ }^{43}$ The MoCA will be assessed at baseline and week 5 by the assessors who will be blinded to the group allocation.

The change in the level of cortisol and melatonin in saliva will be measured before and after the intervention. These levels are known to reflect the circadian rhythm of the human body. The level of cortisol usually surges in the morning, while melatonin release increases at night in normal conditions, and the constant change of these hormones is known to be closely related to arousal and sleep. ${ }^{44}$ We will check whether this type of rhythmical change in these hormones differs before and after the intervention.

To understand the details of the sleep status of the participants, a sleep diary will be provided to each participant. The subjects will be educated to write down their bedtime, final awakening time, sleep latency, number and duration of awakenings, perceived sleep duration, midday nap and use of sleeping pills throughout the study period. ${ }^{46}$

To objectively measure the sleep patterns of the participants, actigraphy will be used in this trial. The candidate participants will wear a watch-type, physical activity monitoring device (ActiGraph wGT3X-BT; MTI Health Services Company, Pensacola, Florida, USA) on their non-dominant wrists for at least 1 week during the screening period. The subjects who meet all eligibility criteria will keep wearing the device during the 4 weeks of the intervention period to record their sleep and activity. Using this method, we can acquire objective data on the participants' sleep quality including the total time in bed, sleep latency, wake after sleep onset, total sleep time and sleep efficiency. ${ }^{23}$

\section{Blinding test and credibility test}

The participants in the EA and sham EA groups will participate in the blinding test and credibility test after the first and last treatments. The purpose of these tests is to check whether the participant blinding was maintained for these groups. The blinding test will ask the participants which type of acupuncture they think has been received, real EA, sham EA or unknown. The credibility test assesses the level of confidence in the treatment they have received. The score of this test ranges from 0 to 6 , where a higher score reflects higher confidence in the treatment. ${ }^{47}$

\section{Safety assessment}

For the safety assessment, the investigators will ask the participants on each visit if they have experienced any adverse events (AEs). Laboratory tests will also be performed at baseline and week 5 to detect AEs. This test includes a complete blood count and differential count, absolute neutrophil count, aspartate aminotransferase, alanine aminotransferase, total bilirubin, blood urea nitrogen, creatinine, albumin, erythrocyte sedimentation rate, TSH, free thyroxine, and human chorionic gonadotropin urine tests (only for women in their childbearing years at the screening visit).

The severity of AEs will be primarily assessed according to the National Cancer Institute Common Terminology Criteria for Adverse Events (NCI-CTCAE) V.4.0. ${ }^{48}$ When the NCI-CTCAE is not applicable, Spilker's three-level criteria will be used instead. ${ }^{49}$ The type of $\mathrm{AE}$ and its severity will be recorded to the CRF regardless of the relationship with the intervention provided in this study. The relationship between the treatment and the $\mathrm{AE}$ will be recorded as definitely related, probably related, possibly related, probably not related, definitely not related or unknown.

\section{Data collection and management}

Data will be collected after acquiring the signed consent from the participants. The collected data will be recorded on the CRF by certificated clinical research coordinators. The whole process of the trial will be regularly monitored to ensure the quality of the trial. Periodic monitoring will confirm that the data in the CRF match the source document and that the procedures of the recruitment, intervention and assessment follow the protocol. 
To guarantee the consistency of the procedures of the intervention and assessment between research centres, a standard operating procedure will be set and provided to the investigators. The practitioners are limited to licensed doctors of Korean medicine who have at least 2 years of clinical experience, and all investigators are required to take training courses for good clinical practice.

To promote participant retention and prevent follow-up loss, the researchers will make phone calls to participants prior to follow-up sessions. We will also inquire the participants failing to attend follow-up or treatment sessions about the reasons for absence and encourage compliance by telephone.

\section{Sample size}

The purpose of this pilot clinical trial is to explore the potential efficacy and safety of EA for insomnia in patients with cancer. Therefore, a prospective sample size calculation was not performed. The result of this study will serve as the basis for sample size calculation for a full-scale trial on this theme in the future. An opinion from an expert in medical statistics recommended that the minimal number of people per group be 12 for a pilot study, ${ }^{50}$ and assuming a dropout rate of 20\%, 15 participants in each group or a total of 45 participants will be deemed appropriate for this research setting.

\section{Statistical analysis}

A full analysis set (FAS), which realises the intention-totreat ideal as closely as possible, ${ }^{51}$ will be the main set for primary analysis. The FAS includes all participants initially allocated to one of the three groups of the study; however, it excludes the data from participants who do not meet the eligibility criteria, who never received the intervention in this study or who never provided any outcome value in this study. A per-protocol (PP) set will be used for sensitivity analysis to compare the results from the FAS. The PP set comprises the participants who completed the trial without major violation of the protocol, with having received at least $70 \%$ of the planned intervention and provided all outcome values.

A two-sided significance level will be set at $5 \%$, and the multiple imputation method will be adopted for missing data. SAS V.9.4 will be used for statistical analysis.

Demographic characteristics and baseline measurements of the variables of each group will be summarised. Continuous data will be expressed as the mean $\pm \mathrm{SD}$. Analysis of variance (ANOVA) or the Kruskal-Wallis test will be performed to compare the continuous data from each group. A 95\% CI can be presented as needed. For dichotomous or categorical data, frequency and percentile will be presented, and the difference between groups will be compared using a $\chi^{2}$ test or the Fisher's exact test.

The mean change of the ISI score from baseline to week 5 is the primary outcome measure of this study. To identify statistically significant differences in the primary outcome measure between groups, two sets of hypotheses are assumed as described below.
Set 1

The null hypothesis $\left(\mathrm{H}_{01}\right)$ : There is no difference in the mean change of the ISI before and after treatment between the EA group and the usual care group.

The alternative hypothesis $\left(\mathrm{H}_{11}\right)$ : There is a difference in the mean change of the ISI before and after treatment between the EA group and the usual care group.

\section{Set 2}

The null hypothesis $\left(\mathrm{H}_{02}\right)$ : There is no difference in the mean change of the ISI before and after treatment between the EA group and the sham EA group.

The alternative hypothesis $\left(\mathrm{H}_{12}\right)$ : There is a difference in the mean change of the ISI before and after treatment between the EA group and the sham EA group.

If $\mathrm{H}_{01}$ is rejected and $\mathrm{H}_{11}$ is adopted in the statistical analysis for set 1 , the test for the hypotheses of set 2 will be verified. However, if $\mathrm{H}_{01}$ is adopted in the test for the set 1 hypotheses, no further validation for the set 2 hypotheses will be required.

To validate the hypotheses, an analysis of covariance will be performed with the baseline value as a covariate and each group as a fixed factor. If there is any significant difference between groups among the demographic characteristics or baseline measurements of the variables, it will be adjusted according to its covariance. The problem of multiple comparison will be solved using the fixed sequence method. ${ }^{52}$

The secondary outcome measures will be analysed via the same methods used for the primary outcome measure analysis. The change of the outcome measures before and after the intervention within a group will be analysed using Student's paired t-test or Wilcoxon signed-rank test. Repeated measures ANOVA with post hoc Dunnett's procedure will be used to validate the differences in the trends per visit.

Subgroup analysis will be conducted to determine whether the severity of insomnia, significant blood deficiency pattern according to a blood deficiency scoring system or level of expectancy to the EA treatment at baseline affects the clinical response to EA. The correlation of the sleep-related variables extracted from an objective source (actigraphy) and a subjective source (patient-reported questionnaires) will be evaluated.

\section{Ethics and dissemination}

Licensed doctors of Korean medicine will obtain informed consent at each research centre. Only the participants who sign the informed consent form will be included in the study. The study protocol has been approved by the institutional review boards (IRBs) of the participating research centres: Daejeon Oriental Hospital of Daejeon University and Dong-eui University Korean Medical Hospital in Busan. The approved protocol was registered with the Clinical Research Information Service (CRIS) of South Korea (CRIS-KCT0002162).

Any modification to the protocol will be reapproved by the IRBs, documented in the online registry of CRIS, 
and reflected in the explanation for the participants. An amended consent form reflecting the revised protocol must be obtained from the participants.

The result of this study will be published in peer-reviewed journals or academic conferences.

\section{DISCUSSION}

Despite the high prevalence and enormous impact of insomnia in patients with cancer, its importance has been overlooked. ${ }^{1}$ The National Cancer Institute and National Comprehensive Cancer Network have strongly recommended evaluation and appropriate treatment of the sleep disturbance of cancer survivors during routine survivorship care. ${ }^{11}$ Nevertheless, it seems that most hospitals and cancer centres are not properly managing the insomnia of patients with cancer. ${ }^{11}$

Insomnia is one of the areas in which acupuncture-related publications have been most commonly focused for the last 20 years. ${ }^{53}$ Previous researches have shown that acupuncture is an effective intervention for insomnia. Acupuncture also appears to be effective for the sleep disorders of patients with cancer, but its effect on the cancer population has been studied relatively less than in general insomniacs. Thus, we expect that the results of this study will contribute to increasing the insufficient evidence in this area of research.

Reviewing previous studies, we found that acupuncture may have only a short-term effect on the insomnia of patients with cancer. Mao et al reported that the sleep quality of the EA group was significantly better than that of control group immediately after an 8-week intervention period, but this difference between groups disappeared after 4 weeks. ${ }^{54}$ Another study by Otte $e t$ al also showed a similar pattern that the effect of a 2-week acupuncture treatment vanished after a 4 -week follow-up period. ${ }^{28}$

However, according to the opinion of Choi et al, the effect of acupuncture seems to be strengthened over time. ${ }^{20}$ These authors found that acupuncture was as good as conventional therapy and became better at follow-up visits 3 weeks after final treatment. ${ }^{20}$ We will record the trend change of the effect of EA over time by assessing the response of the participants at baseline; week 3, a midpoint of the 4-week intervention; week 5 , immediately after the end of the final intervention; and week 9 , the final follow-up assessment. We expect the results of this study to contribute to solving the disagreement among the existing studies on the timing and duration of the effect of acupuncture on sleep disturbance in patients with cancer.

In addition to time duration, there are many factors that can influence the effectiveness of acupuncture. Among them, we will try to determine whether a certain pattern identified according to the theory of Korean traditional medicine, blood deficiency, is an important variable that can affect the response to acupuncture. Blood deficiency is one of the main patterns of insomnia in Korean traditional medicine, and this study will investigate how many patients are categorised with a blood deficiency pattern using a blood deficiency scoring system ${ }^{55}$ and whether their treatment response is different from that of the non-blood deficiency group.

In insomnia clinical trials, the placebo effect is very commonly observed. ${ }^{56}$ The characteristics of insomniathat it is intermittent and tends to maintain or even reinforce the therapeutic effect of specific treatment over long periods-seem to explain such a placebo effect. ${ }^{56}$ This is the reason why the inclusion of a placebo control is generally recommended in insomnia clinical trials. ${ }^{56}$ Thus, this study also included a sham EA group as a placebo control.

The sham EA group will receive sham acupuncture therapy at non-acupoints unrelated to insomnia using a Streitberger acupuncture needle. This sham needle was devised by Streitberger and Kleinhenz in $1998 .{ }^{57}$ Its appearance is the same as real acupuncture needles, but the needle does not penetrate the skin. The suitability of this device as a placebo comparator for acupuncture needles has been validated, ${ }^{57}$ and it has been used as a control intervention in several acupuncture clinical studies. ${ }^{58}$

The Streitberger acupuncture needle is one of the two most commonly used and commercially available placebo devices in acupuncture clinical studies along with the Park sham device. ${ }^{58}$ Nevertheless, approximately half of the clinical studies using these sham devices have concluded that the effects of real acupuncture and sham needles were not significantly different. ${ }^{58}$ Generally, a placebo pill is used on the assumption that it has no pharmacological effect and is harmless. ${ }^{59}$ However, it has been shown in many studies to date that sham acupuncture needles seem to have a significant physiological effect. ${ }^{59}$ Thus, the development of a new placebo device as a perfect comparator for real acupuncture that has no physiologic effects while maintaining patient blinding is still a significant challenge for acupuncture researchers. However, we currently have no choice but to use one of these available sham devices while considering their limitations.

It is known that the proportion of the placebo effect is not inconsiderable in acupuncture treatment. ${ }^{59}$ Moreover, as we have seen, patients with insomnia tend to respond strongly to placebo treatment. In addition, the response to sham acupuncture such as the skin sensation of pressure or tingling may be akin to the response to acupressure, which is also known to be effective for insomnia. ${ }^{16}$ Thus, a study design that directly compares acupuncture and sham acupuncture is likely to produce a false negative result, failing to detect the whole characteristic effect of acupuncture. ${ }^{59}$ Therefore, we designed a three-arm parallel study including the usual care group as well as a sham control group.

The sample size of this pilot study is 15 per each group, which may be underpowered to confirm the hypothesis of the study. Further study will be required to draw a definitive conclusion of the effectiveness of EA for improving insomnia in patients with cancer. The result of this 
preliminary study will become a basis to design a full-scale RCT to conclude this issue.

The strength of this study is that it will acquire objective outcomes reflecting sleep quality and the related circadian rhythm. First, we will obtain both subjective and objective outcomes showing sleep quality using patient-reported questionnaires and actigraphy. Most of the acupuncture studies on insomnia have used only patient-reported outcomes; furthermore, even a small number of studies using objective outcomes such as polysomnography or actigraphy failed to show consistent results on the effect of acupuncture for each outcome. ${ }^{30-34}$ Based on the results of this study, we expect that we will be able to compare the difference between the subjective and objective outcomes and contribute to solving the conflict on this issue. Only actigraphy is used for objective outcome measures at this time; however, polysomnography, a gold standard method for insomnia diagnosis, should be included in future research.

Second, the difference in the levels of cortisol and melatonin in saliva between the groups will be compared as biomarkers reflecting the circadian rhythm of the body that affects the pattern of sleep. It is known that acupuncture modulates various neurotransmitters and hormones such as endorphin, serotonin, norepinephrine, cortisol and melatonin. ${ }^{445}$ Among them, changes of the cortisol surge in the morning or melatonin increase at nighttime are known to be closely related to arousal and sleep. ${ }^{44}$ Previous studies have reported that acupuncture can improve insomnia by affecting the pattern of cortisol $^{6061}$ or melatonin ${ }^{27}$ release. Based on these results, we will investigate the changes in salivary cortisol and melatonin in addition to the sleep quality of the participants.

\section{Trial status}

This trial is currently recruiting participants.

Contributors MK and J-HK conceived the study. MK drafted the protocol. J-EK, $\mathrm{H}-\mathrm{YL}, \mathrm{A}-\mathrm{RK}$ and $\mathrm{H}-\mathrm{JP}$ participated in the design of the study and contributed to the refinement of the protocol. 0-JK was responsible for the statistical design of the study. B-KK and JHC provided clinical advice and made critical revisions. $\mathrm{J}$-HK is a principal investigator of the study and has the final responsibility for the decision to submit this manuscript for publication. All authors approved the final manuscript.

Funding This work was supported by the Korea Institute of Oriental Medicine (grant number K16122 \& K17122).

Competing interests None declared.

Ethics approval Daejeon Oriental Hospital of Daejeon University IRB (djomc-140-1); Dong-eui University Korean Medical Hospital IRB (2016-04).

Provenance and peer review Not commissioned; externally peer reviewed.

Data sharing statement Requested data for public purpose or research transparency will be provided via the corresponding author.

Open Access This is an Open Access article distributed in accordance with the Creative Commons Attribution Non Commercial (CC BY-NC 4.0) license, which permits others to distribute, remix, adapt, build upon this work non-commercially, and license their derivative works on different terms, provided the original work is properly cited and the use is non-commercial. See: http://creativecommons.org/ licenses/by-nc/4.0/ (c) Article author(s) (or their employer(s) unless otherwise stated in the text of the article) 2017. All rights reserved. No commercial use is permitted unless otherwise expressly granted.

\section{REFERENCES}

1. Savard J, Morin CM. Insomnia in the context of cancer: a review of a neglected problem. J Clin Oncol 2001;19:895-908.

2. Beck SL, Berger AM, Barsevick AM, et al. Sleep quality after initial chemotherapy for breast Cancer. Support Care Cancer 2010;18:679-89.

3. Fiorentino L, Ancoli-Israel S. Sleep dysfunction in patients with cancer. Curr Treat Options Neurol 2007;9:337-46.

4. Lis CG, Gupta D, Grutsch JF. The relationship between insomnia and patient satisfaction with quality of life in cancer. Support Care Cancer 2008;16:261-6.

5. Bower JE, Ganz PA, Irwin MR, et al. Inflammation and behavioral symptoms after breast cancer treatment: do fatigue, depression, and sleep disturbance share a common underlying mechanism? J Clin Oncol 2011;29:3517-22.

6. Sharma N, Hansen $\mathrm{CH}, \mathrm{O}^{\prime}$ Connor M, et al. Sleep problems in cancer patients: prevalence and association with distress and pain. Psychooncology 2012;21:1003-9.

7. Jean-Pierre P, Grandner MA, Garland SN, et al. Self-reported memory problems in adult-onset cancer survivors: effects of cardiovascular disease and insomnia. Sleep Med 2015;16:845-9.

8. Payne J, Piper B, Rabinowitz I, et al. Biomarkers, fatigue, sleep, and depressive symptoms in women with breast cancer: a pilot study. Oncol Nurs Forum 2006;33:775-83.

9. Innominato PF, Focan C, Gorlia T, et al. Circadian rhythm in rest and activity: a biological correlate of quality of life and a predictor of survival in patients with metastatic colorectal cancer. Cancer Res 2009;69:4700-7.

10. Cash E, Sephton SE, Chagpar AB, et al. Circadian disruption and biomarkers of tumor progression in breast cancer patients awaiting surgery. Brain Behav Immun 2015;48:102-14.

11. Zhou ES, Partridge AH, Syrjala KL, et al. Evaluation and treatment of insomnia in adult cancer survivorship programs. J Cancer Surviv 2017;11:74-9.

12. Garland SN, Gehrman P, Barg FK, et al. CHoosing Options for Insomnia in Cancer Effectively (CHOICE): Design of a patient centered comparative effectiveness trial of acupuncture and cognitive behavior therapy for insomnia. Contemp Clin Trials 2016;47:349-55.

13. Moore TA, Berger AM, Dizona P. Sleep aid use during and following breast cancer adjuvant chemotherapy. Psychooncology 2011;20:321-5

14. Espie CA, MacMahon KM, Kelly HL, et al. Randomized clinical effectiveness trial of nurse-administered small-group cognitive behavior therapy for persistent insomnia in general practice. Sleep 2007;30:574-84.

15. Cao H, Pan X, Li H, et al. Acupuncture for treatment of insomnia: a systematic review of randomized controlled trials. $J$ Altern Complement Med 2009;15:1171-86.

16. Cheuk DK, Yeung WF, Chung K, et al. Acupuncture for insomnia. Cochrane Database Syst Rev 2012;12:CD005472.

17. Shergis JL, Ni X, Jackson ML, et al. A systematic review of acupuncture for sleep quality in people with insomnia. Complement Ther Med 2016;26:11-20.

18. Garcia MK, Cohen L, Spano M, et al. Inpatient Acupuncture at a Major Cancer Center. Integr Cancer Ther 2016.

19. Garland SN, Xie SX, Li Q, et al. Comparative effectiveness of electroacupuncture versus gabapentin for sleep disturbances in breast Cancer survivors with hot flashes: a randomized trial. Menopause 2017;24:517-523.

20. Choi TY, Kim JI, Lim HJ, et al. Acupuncture for managing CancerRelated Insomnia: a Systematic Review of Randomized clinical trials. Integr Cancer Ther 2017;16:135-46.

21. Krystal AD, Edinger JD. Measuring sleep quality. Sleep Med 2008;9:S10-S17.

22. Jacobs EA, Reynolds CF, Kupfer DJ, et al. The role of polysomnography in the differential diagnosis of chronic insomnia. Am J Psychiatry 1988;145:346-9.

23. Sadeh A, Hauri PJ, Kripke DF, et al. The role of actigraphy in the evaluation of sleep disorders. Sleep 1995;18:288-302.

24. Zhang L, Zhao ZX. Objective and subjective measures for sleep disorders. Neurosci Bull 2007;23:236-40.

25. Hachul H, Garcia TK, Maciel AL, et al. Acupuncture improves sleep in postmenopause in a randomized, double-blind, placebo-controlled study. Climacteric 2013;16:36-40. 
26. Ruan JW, Wang CH, Liao XX, et al. Electroacupuncture treatment of chronic insomniacs. Chin Med J 2009;122:2869-73.

27. Spence DW, Kayumov L, Chen A, et al. Acupuncture increases nocturnal melatonin secretion and reduces insomnia and anxiety: a preliminary report. J Neuropsychiatry Clin Neurosci 2004;16:19-28.

28. Otte JL, Carpenter JS, Zhong X, et al. Feasibility study of acupuncture for reducing sleep disturbances and hot flashes in postmenopausal breast cancer survivors. Clin Nurse Spec 2011;25:228-36.

29. Yeung WF, Chung KF, Zhang SP, et al. Electroacupuncture for primary insomnia: a randomized controlled trial. Sleep 2009;32:1039-47.

30. Hwang EH. [Effects of hand acupuncture therapy on sleep quality in sleep disrupted adults: verification by polysomnography and cerebral blood flow test]. Taehan Kanho Hakhoe Chi 2007;37:1108-18.

31. Kaiser-Pagliarini TG, Hachul HC, Maciel AL, et al. 064 acupuncture in insomnia and its consequences in postmenopausal women. Sleep Med 2009;10:S18.

32. Chung KF, Yeung WF, Zhang SP, et al. Acupuncture for persistent insomnia associated with major depressive disorder: a randomised controlled trial. Hong Kong Med J 2016;22(Suppl2):S9-14.

33. Chung KF, Yeung WF, Yu YM, et al. Acupuncture for residual insomnia associated with major depressive disorder: a placebo- and sham-controlled, subject- and assessor-blind, randomized trial. $J$ Clin Psychiatry 2015;76:e752-60.

34. Yeung WF, Chung KF, Tso KC, et al. Electroacupuncture for residual insomnia associated with major depressive disorder: a randomized controlled trial. Sleep 2011;34:807-15.

35. Morin CM, Belleville G, Bélanger L, et al. The Insomnia Severity Index: psychometric indicators to detect insomnia cases and evaluate treatment response. Sleep 2011;34:601-8.

36. Cho YW, Song ML, Morin CM. Validation of a Korean version of the insomnia severity index. J Clin Neurol 2014;10:210-5.

37. Buysse DJ, Reynolds CF, Monk TH, et al. The Pittsburgh Sleep Quality Index: a new instrument for psychiatric practice and research. Psychiatry Res 1989;28:193-213.

38. Sohn SI, Kim DH, Lee MY, et al. The reliability and validity of the korean version of the Pittsburgh Sleep Quality Index. Sleep Breath 2012;16:803-12.

39. Yellen SB, Cella DF, Webster K, et al. Measuring fatigue and other anemia-related symptoms with the functional Assessment of Cancer therapy (FACT) measurement system. J Pain Symptom Manage 1997:13:63-74

40. Lee EH, Chun M, Kang S, et al. Validation of the Functional Assessment of Cancer Therapy-General (FACT-G) scale for measuring the health-related quality of life in Korean women with breast cancer. Jpn J Clin Oncol 2004;34:393-9.

41. Jeong JS, Ryu BH, Kim JS, et al. Bojungikki-tang for cancerrelated fatigue: a pilot randomized clinical trial. Integr Cancer Ther 2010;9:331-8.

42. Arcuri GG, Palladini L, Dumas G, et al. Exploring the measurement properties of the Montreal Cognitive Assessment in a population of people with Cancer. Support Care Cancer 2015;23:2779-87.

43. Lee JY, Dong Woo Lee, Cho SJ, et al. Brief screening for mild cognitive impairment in elderly outpatient clinic: validation of the
Korean version of the Montreal Cognitive Assessment. $J$ Geriatr Psychiatry Neurol 2008;21:104-10.

44. Huang W, Kutner N, Bliwise DL. Autonomic activation in insomnia: the case for acupuncture. J Clin Sleep Med 2011;7:95-102.

45. Zhao K. Acupuncture for the treatment of insomnia. Int Rev Neurobiol 2013;111:217-34.

46. Carney CE, Buysse DJ, Ancoli-Israel S, et al. The consensus sleep diary: standardizing prospective sleep self-monitoring. Sleep 2012;35:287-302.

47. Devilly GJ, Borkovec TD. Psychometric properties of the credibility/expectancy questionnaire. J Behav Ther Exp Psychiatry 2000;31:73-86

48. US department of Health and Human Services. National Cancerlnstitute: National Institutes of Health (2009). Common terminology criteria for adverse events (CTCAE) version 4.0. National Institutes of Health, 2009. https://evs.nci.nih.gov/ftp1/CTCAE/ CTCAE_4.03_2010-06-14_QuickReference_5x7.pdf.

49. Spilker B. Quality of life and pharmacoeconomics in clinical trials. 2nd ed. Philadelphia: Lippencott-Raven, 1995.

50. Julious SA. Sample size of 12 per group rule of thumb for a pilot study. Pharm Stat 2005;4:287-91.

51. European Medicines Agency. ICHTopicE9. Statistical principlesforclinical trials. http://www.ema.europa.eu/docs/en GB/ document_library/Scientific_guideline/2009/09/WC500002928.pdf. (accessed 19 Jan 2017).

52. Wiens BL. A fixed sequence Bonferroni procedure for testing multiple endpoints. Pharm Stat 2003;2:211-5.

53. Ma Y, Dong M, Zhou K, et al. Publication trends in Acupuncture Research: a 20-Year Bibliometric analysis based on PubMed. PLoS One 2016;11:e0168123.

54. Mao JJ, Farrar JT, Bruner D, et al. Electroacupuncture for fatigue, sleep, and psychological distress in breast Cancer patients with aromatase inhibitor-related arthralgia: a randomized trial. Cancer 2014;120:3744-51.

55. Terasawa KT. Kampo diagnosis of western medical doctor. Seoul: Jipmundang, 1998.

56. Perlis ML, McCall WV, Jungquist CR, et al. Placebo effects in primary insomnia. Sleep Med Rev 2005;9:381-9.

57. Kleinhenz J, Streitberger K, Windeler J, et al. Randomised clinical trial comparing the effects of acupuncture and a newly designed placebo needle in rotator cuff tendinitis. Pain 1999;83:235-41.

58. Zhang CS, Tan HY, Zhang GS, et al. Placebo Devices as Effective Control methods in Acupuncture clinical trials: a systematic review. PLoS One 2015;10:e0140825.

59. Paterson C, Dieppe P. Characteristic and incidental (placebo) effects in complex interventions such as acupuncture. BMJ 2005;330:1202-5.

60. Akimoto T, Nakahori C, Aizawa K, et al. Acupuncture and responses of immunologic and endocrine markers during competition. Med Sci Sports Exerc 2003;35:1296-302.

61. Liu S, Zhou W, Ruan X, et al. Activation of the hypothalamus characterizes the response to acupuncture stimulation in heroin addicts. Neurosci Lett 2007;421:203-8. 